\title{
Temporal Resources for Global Quantum Computing Architectures
}

\author{
Juan D. Jaramillo* and John H. Reina ${ }^{\dagger}$ \\ Departamento de Física, Universidad del Valle, Apartado Aéreo 25360, Cali, Colombia \\ (Received on 3 April, 2008)
}

\begin{abstract}
Using the methods for optimal simulation of quantum logic gates, we perform a quantitative estimation of the time resources involved in the execution of universal gate sets for the case of three representative models of quantum computation based on global control. The importance of such models stems from the solution to the problem of experimentally addressing and locally manipulating the qubits in a given quantum register. The numerical estimation of the temporal efficiency for each model is performed by assuming that the qubits in the register can be coupled to each other via the Ising and the Förster interactions. Finally, we discuss the feasibility of the physical realization of such architectures under quantum error correction conditions.
\end{abstract}

Keywords: Quantum control; Quantum computation; Quantum error correction

\section{INTRODUCTION}

Quantum computation stands as one of the outstanding innovations of current technologies. As Feynman pointed out almost five decades ago [1], the development of smaller electronic devices ultimately leads to a consideration of quantum mechanical effects in electronic and computer designs [2]. Moreover, it became clear after the works of Deutsch [3] and Shor [4] that quantum mechanics could provide the means for a radical new way of computing, allowing the computation of many intractable computational problems in "classical machines" (computers running under "classical" phenomenology), such as factoring of large numbers [4] or the exact simulation of large quantum systems [5]. Aside from the usual model of quantum circuits for quantum computation (QC) [3] there are other models under current investigation, such as one-way QC [6], adiabatic QC [7], quantum cellular automata [8], and others. Although these models are equivalent in the sense that they can simulate each other with polynomial slowdown in its time and space resources, when it comes to physical realizations, polynomial differences between models become a relevant issue regarding error robusticity [9].

A problem of central importance is the necessity to characterize the efficiency of such models in different computational problems and for different physical systems. Qubit chains associated with two level quantum systems interacting with their nearest neighbours are a common array for many QC models. In these arrays, the input information is stored along with the subsequent evolution of the system. In order to perform any quantum computation on the register, it is sufficient to be able to realize a universal set of one and two-qubit quantum gates [10]. There are two main mechanisms for the implementation of localized multi-qubit gates in a quantum register: the traditional 'local' control (LC) and the so-called 'global' control (GC). In LC quantum computation (LCQC), an induced evolution in the system requires the direct individual localization of the computational qubits. A problem common to such

\footnotetext{
*Electronic address: judi jasa@univalle.edu.co

${ }^{\dagger}$ Electronic address: jhreina@univalle.edu.co
}

a strategy is the difficulty in its physical implementation, especially at scales where the manipulation invokes more than a few qubits register. By contrast, in GC quantum computing (GCQC) the induced evolution of the system doesn't require direct individual localization of the computational qubits to be targeted by the logic gates; the model manages to induce a localized gate by using the instructions stored in the register's initial configuration. Thus, from the viewpoint of quantum information technologies, it may be more promising to be able to realize unitary manipulations within the quantum register in a global fashion.

In this work, we evaluate the additional temporal resources required for the execution of three relevant architectures for GCQC [11-13]. Our results are based on a precise determination of temporal resources by means of techniques based on time-optimal simulation of quantum gates $[14,15]$. Moreover, we also estimate the spatial resources involved in the conditional dynamics performance and discuss the feasibility of such architectures for implementing quantum error correction over the computational and the auxiliary qubits considered in Refs. [11-13].

\section{TIME-OPTIMAL SIMULATION OF QUANTUM GATES}

Calculation of optimal times for the execution of quantum gates depends specifically on the form of the interacting Hamiltonian between qubits. The optimal time execution of an $n$-qubits gate $\mathcal{U}$, using a Hamiltonian $\mathcal{H}$, is defined as the minimal time $T$ executing $\mathcal{U}$ in the controllable system [16]:

$$
\dot{U}(t)=-i \mathcal{H}(t) U(t) ; U(0)=I ; U(T)=\mathcal{U},
$$

where $U(t)$ belongs to the special unitary group $S U\left(2^{n}\right), I$ is the group's identity element, $T$ is the time it takes to go from $I$ to $\mathcal{U}$ along $S U\left(2^{n}\right)$, and

$$
\mathcal{H}(t) \equiv \mathcal{H}_{0}+\mathcal{H}_{C t r l}(t),
$$

is a curve in the space of Hermitic matrices of dimension $n$, decomposed as a sum of a time independent matrix $\mathcal{H}_{0}$ and a dynamically controllable term $\mathcal{H}_{\text {Ctrl }}(t)$. A special case of these systems, termed local control systems, occurs when $\mathcal{H}_{0}$ 
is associated to a qubit-qubit interacting Hamiltonian (e.g., Eq. (6)) and the controllable term $\mathcal{H}_{\text {Ctrl }}(t)$ to a local (one qubit) Hamiltonian, which could be physically realized, e.g., through the coupling with a controllable external magnetic field. In this work, we apply the techniques introduced in Refs. $[14,15]$ for treating quantum computer systems to the case of globally controlled systems. A central consideration here is the fast control limit $[14,15]$, by which the execution time of local Hamiltonians is considered "arbitrarily fast", an assumption based on the experimental consideration that the strength of the coupling between the qubit and the controllable external field can be raised high enough as to consider negligible the time of one qubit rotations in comparison with the time induced by the qubit-qubit interactions.

Formally, this means that if we let $G=S U\left(2^{n}\right)$, and $K$ be the subgroup of $G$ associated to local unitary operations [16], i.e., unitaries with the form $\exp \left(-i \mathcal{H}_{C t r l}\right)$, then

1. The execution time for $\mathcal{U}$ is equivalent to that for $K \mathcal{U}$ and $\mathcal{U} K . \mathcal{U}$ is said to be locally equivalent to $\tilde{\mathcal{U}}$ if there exist $k_{1}, k_{2} \in K$ such that $\mathcal{U}=k_{1} \tilde{\mathcal{U}} k_{2}$.

2. Finding the optimal time for simulating a quantum gate $\mathcal{U} \in G$ is equivalent to finding the geodesic between $I$ and $\mathcal{U}$ in the coset space $G / K$.

Here, we focus on the results for two and three-qubit gates, which are the core of the considered GCQC protocols. Optimal time solutions for locally controlled systems of two-qubit gates have already been reported [14]. The conditions for an optimal simulation of a quantum gate $\mathcal{U}$ under an interacting Hamiltonian $H_{0}$ can be reduced to finding the minimum time $t$ [17], such that

$$
\vec{\alpha} \prec_{S} \vec{\beta} t
$$

where $\vec{\alpha}$ and $\vec{\beta}$ are coefficients characterizing the unitaries $\exp \left(-i \sum \alpha_{i} \sigma_{i} \otimes \sigma_{i}\right)$ and $\exp \left(-i \sum \beta_{i} \sigma_{i} \otimes \sigma_{i}\right)$, which are locally equivalent to $\mathcal{U}$ and $\exp \left(-i H_{0}\right)$ respectively, and the $\sigma_{i}$ 's denote the Pauli matrices. The special majorization relation $\prec_{S}$ is established in direct relation to the normal majorization relation between the vectors $\vec{\lambda}$ and $\vec{v}$ associated to the eigenvalues of $\exp \left(-i \sum \alpha_{i} \sigma_{i} \otimes \sigma_{i}\right)$ and $\exp \left(-i \sum \beta_{i} \sigma_{i} \otimes \sigma_{i}\right)$, respectively. It is important to note that the previous analytic solution relies on the fact that $S U(4) /(S U(2) \times S U(2))$ is a Riemannian symmetric space, which implies the existence of an abelian subalgebra. This assures the characterization of locally equivalent operators based on unitaries of the generic form $\exp \left(-i \sum \alpha_{i} \sigma_{i} \otimes \sigma_{i}\right)$. Regarding threequbit gates, optimal simulation results have only been found under specific situations $[15,18]$. Here, the previous approach is no longer valid: geodesics have to be solved in $S U(8) /(S U(2) \times S U(2))$, which is not Riemannian symmetric. Important quantum gates such as the CNOT and SWAP between the first and the third qubit have been solved by a combination of analytical and numerical techniques $[15,18]$. Other quantum gates, such as the ones we apply in this work, are indirectly addressed by decomposition into already solved quantum gates.

\section{MODELS FOR QUANTUM COMPUTATION BASED ON GLOBAL CONTROL}

The GCQC models are arrays of two level quantum systems interacting, in first approximation, with their nearest neighbours. There exists a finite number of "qubit species" distributed in an alternate manner within the arrays, as shown schematically in Fig. 1(a). Here, each species can be collectively manipulated in an independent way. An example of a physical realization of such architectures is a linear array of coupled quantum systems which has associated a periodic and finite set of frequencies $\left(\omega_{A}, \omega_{B}, \omega_{A}, \cdots\right)$ that can be addressed through resonant radiofrequency (RF) pulses. Between the 'computational' qubits, those effectively involved in the computation, there are auxiliary qubits or "ancillae", with purely operative functions which are initialized in the computational state $|0\rangle^{\otimes m}$. Besides the ancillae, there is a "special qubit", the "control unit", whose role it is to localize and 'transport' information between the computational qubits. One qubit gates, for example, are performed in two steps: first, the control unit is taken near enough to the computational qubit to be modified, and then the desired gate is performed over the computational qubit, as a controlled gate, where the control unit acts as the control qubit.

We next introduce the three architectures and the respective operative protocols that we use in our calculations.

i) Model $1(B M 1)$. Proposed by Benjamin [11], this is one of the simplest models for GCQC; it consists of two species of physical qubits, $A$ and $B$, as shown in Fig. 1 . The computational qubits are encoded in physical qubits $(|0\rangle \equiv|\uparrow\rangle$ and $|1\rangle \equiv|\downarrow\rangle$ ) belonging exclusively to a given species with the exception of the "special qubit" or "control unit", which is initialized in a different species at the computational state $|1\rangle$. There are arrays of three and five ancillae qubits alternately distributed between the computational qubits (see Fig. 1(b)). The generic Hamiltonian for this system is given by $H=\sum_{j=1}^{n} H_{j}^{s}+\sum_{j=1}^{n} H_{j, j+1}^{\mathrm{int}}$, where the first term is associated with the individual qubit energy and the latter with the interaction energy between neighbouring qubits. The particular characteristics of the system reduce the total Hamiltonian to the form [11]:

$$
\begin{aligned}
H_{2 j} & =H^{A}, \\
H_{2 j+1} & =H^{B}, \\
H_{2 j, 2 j+1} & =H^{A B}, \\
H_{2 j+1,2 j} & =H^{B A} .
\end{aligned}
$$

This model works as long as the following conditions are fulfilled: a) it has to be possible to control the supression of the interaction process due to $H^{B A}$, in a way that the system reduces to a set of pairs A-B interacting identically through $H^{A B}$; b) any quantum gate must be able to be realized, in the A-B pairs, through the manipulation of the remaining terms: $H^{A}, H^{B}$ and $H^{A B}$; c) as in the requirements a) and b), but this time supressing the interaction $H^{A B}$. These conditions may, however, pose a challenge from an experimental point of 
view [19]. To alleviate such difficulties, a strategy that incorporates a third energy level as part of one of the qubit species (a "barrier"), has been put forward in Ref. [19], at the cost of increasing the number of species in the array. (a)

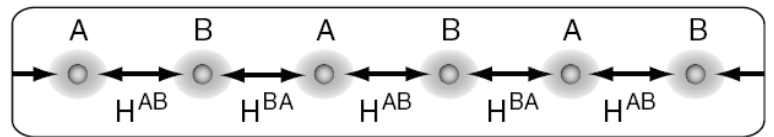

(b)

A $B$ A $B \cdots$ (c)

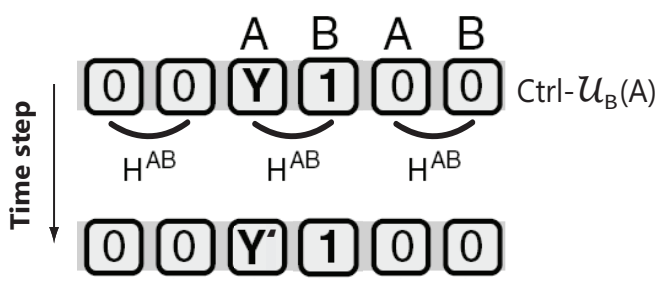

FIG. 1: (a) Schematic of a periodic array of two types of qubits ( $A$ and $B$ ), present in the architectures $B M 1$ and $B M 2$ (see text) for quantum computation based on global control. (b) Array of ancillae and computational qubits in architecture $B M 1$. (c) One qubit gate $\mathcal{U}$ acting selectively on qubit $\mathbf{Y}$. The gate is indirectly performed using the controlled quantum gate $\operatorname{Ctrl}_{B}(A)$, which uses the $H^{A B}$ interaction Hamiltonian. The "control unit" acts as the control qubit and assures the localized action of $\mathcal{U}$. Adapted from Ref. [11].

As an illustration, in Fig. 1(c) we show how to perform a one computational qubit gate: the control unit is located at an adjacent cell from the target qubit $(\mathbf{Y})$. Making sure that the interacting Hamiltonian between the target and the control unit is turned on $\left(H^{A B}\right)$, any arbitrary controlled gate $(C t r l-\mathcal{U})$ can be performed by means of using the species where the control unit is located as the control qubit and the species where the target computational qubit is located as the target.

ii) Model 2 (BM2). This model actually precedes $B M 1$ [12]. It has the same Hamiltonian configuration specified in Eq. (4). Unlike BM1, this model doesn't require the ability to independently control the interacting Hamiltonians, $H^{A B}$ and $H^{B A}$. This benefit doesn't come free; in this case the computational qubits are encoded in four physical qubits, as follows: $|0\rangle \equiv|\uparrow \uparrow \downarrow \downarrow\rangle$ and $|1\rangle \equiv|\downarrow \downarrow \uparrow \uparrow\rangle$. Between every encoded computational qubit there are four ancillae qubits. The "control unit" is also encoded, but in a different configuration: $|\uparrow \uparrow \downarrow \downarrow \uparrow \uparrow\rangle$ and $|\uparrow \uparrow \uparrow \uparrow \uparrow \uparrow\rangle$, , representing the computational states $|1\rangle$ and $|0\rangle$, respectively. The complete array is shown in Fig. 2(a). The operational gates which, applied sequentially, perform any computational gate, are symmetric three qubit gates of the generic form

$$
\begin{aligned}
M\left(u_{00}, u_{01}, u_{10}, u_{11}\right)= & |00\rangle\left\langle 00\left|\otimes u_{00}+\right| 01\right\rangle\langle 01| \otimes u_{01}+ \\
& |10\rangle\left\langle 10\left|\otimes u_{10}+\right| 11\right\rangle\langle 11| \otimes u_{11},
\end{aligned}
$$

where $M$ acts simultaneously over every physical qubit of a given species and the symmetric condition $u_{01}=u_{10}$ is fulfilled. This condition is compatible with the fact that neighbouring qubits are of the same species and therefore only symmetric gates are physically feasible.
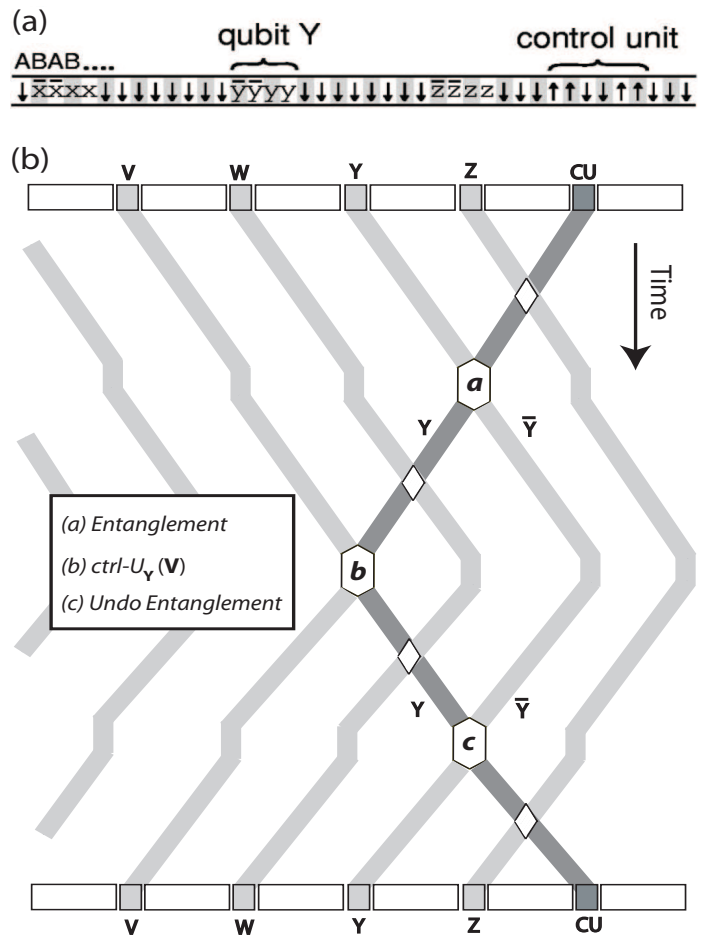

FIG. 2: (a) Array of ancillae and computational qubits in architecture $B M 2$. (b) Heuristic protocol for two-qubit ( $\mathbf{V}$ and $\mathbf{Y}$ ) computational gates in models $B M 1$ and $B M 2$. Computational qubits are in light gray and distinguished by letters. The white space between computational qubits corresponds to ancillae qubits, and the line in dark grey corresponds to the control unit path. Adapted from Ref. [12].

Both models, BM1 and BM2, perform computational two-qubit controlled gates using the control unit as the carrier of information from the "control" qubit to the "target" qubit. This is done through the entanglement between the control unit and the control qubit, as illustrated in Fig. 2(b). A calculation made in the present work shows that in order to perform a more general two-qubit gate under the former protocol, two controlled gates are required; a fact that triples the computational time. To see why this is so, consider the case where a general two-qubit gate is performed through the scheme depicted in Fig. 2(b). In this case, the state of the control unit (dark gray) approaching step (c) may carry information about qubit state $\mathbf{V}$, given the action of the general two-qubit gate performed at step (b). This disables the possibility of recovering the original localized qubit state Y through the step (c) where the entanglement is destroyed.

iii) Model 3 (LM3). Introduced by Lloyd in 1993 [13], this model is perhaps the first proposal for GCQC. It has three different species distributed in a periodic array $A B C A B C A B C$. 
Here, there's no need to control interacting Hamiltonians and computational qubits are encoded in physical qubits belonging exclusively to a given species, just as in $B M 1$. There are two ancillae qubits between the computational qubits.

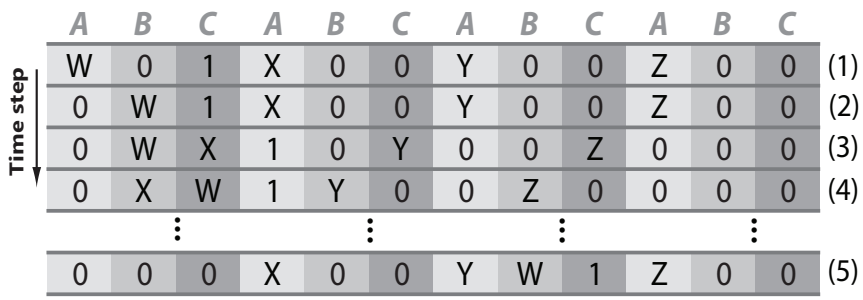

FIG. 3: Protocol for a two-qubit quantum gate between arbitrary computational qubits $\mathbf{W}$ and $\mathbf{Z}$, in architecture $L M 3$. Every computational qubit is initiated at qubit species $A$. (1) The control unit is located at the neighbourhood of $\mathbf{W}$, in this case $\mathbf{W}$ is at species $A$, while the control unit is at species $C$. (2) A controlled SWAP gate between species $A$ and $B$ is applied by using qubits at species $C$ as the control qubits. Given the location of the control unit, only the computational qubit $\mathbf{W}$ will be transferred to species $B$. (3) A $S W A P$ gate is applied between species $A$ and $C$. (4) A SWAP gate is applied between species $B$ and $C$. (5) Steps 2, 3, and 4 are applied again. The register is ready to implement a two-qubit gate between computational qubits $\mathbf{W}$ and $\mathbf{Z}$ using the control unit. This is done after applying a controlled two-qubit gate between species $A$ and $B$, where the species $C$ acts as the control qubit. Any other operation can be reverted so that the modified computational qubits $\mathbf{W}$ and $\mathbf{Z}$ can go to their initial locations in the register.

The operative gates that add up to perform computational gates are non-symmetric three qubit gates of the generic form given by Eq. (5). Unlike the models above, two-qubit gates are performed by the transportation of one of the computational qubits to a position adjacent to the second computational qubit involved in the two-qubit gate. This process, illustrated in Fig. 4, is performed by using the control unit to exclusively transport the first computational qubit $(\mathbf{W})$ through the register. Once the control unit and the two computational qubits $(\mathbf{W}$ and $\mathbf{Z}$ ) are all in the same neighbourhood, $A B C$, the system is ready to apply any two-qubit computational gate, where only the former computational qubits are involved. This is assured given that the control unit acts as a control qubit for the action of the two-qubit gate over the former computational qubits.

\section{RESULTS AND DISCUSSION}

For the evaluation of the growth of parameters regarding the number of computational qubits, the number of ancillae qubits between the computational qubits and the number of physical qubits required to codify a computational qubit were taken into account. The evaluation of the time performance of computational gates in the three architectures is based on the results of optimal simulation of quantum gates discussed in Section $2[14,15]$. In a general picture, we consider quantum registers under global control, for which an anisotropic
Heisenberg interaction mediates the process of non-local gating:

$$
\mathcal{H}_{\text {int }}=\sum_{i} J_{X Y}\left(\sigma_{x}^{i} \otimes \sigma_{x}^{i+1}+\sigma_{y}^{i} \otimes \sigma_{y}^{i+1}\right)+J_{Z} \sigma_{z}^{i} \otimes \sigma_{z}^{i+1}
$$

which contains both the planar $X Y$ or Förster interaction $\left(J_{Z}=\right.$ $0)$, and the isotropic Heisenberg interaction $\left(J_{Z}=J_{X Y}\right)$ as limits.

\subsection{Space and time scaling factors}

We first consider a register for which there is an effective Ising interaction $\mathcal{H}_{\text {int }} \equiv \mathcal{H}_{\text {sing }}$ between neighbouring qubits: $J_{Z} \equiv J, J_{X Y}=0$. Spatial scaling analysis is concerned with the relation between the number of computational qubits, $N$, and the number of physical qubits, $N_{p}$. The three models scale linearly with respect to the total number of computational qubits, and the proportionality factor, $k$, between $N_{p}$ and $N$ is the unique parameter characterizing the spatial scaling in the three architecures.

\begin{tabular}{|l|l|}
\hline Model LM3 & $3 N$ \\
\hline Model BM2 & $8 N$ \\
\hline Model BM1 & $4 N$ \\
\hline
\end{tabular}

TABLE I: Average spatial scaling for the three architectures as function of the number, $N$, of computational qubits.

Spatial scaling factors, without taking into account quantum error correction nor parallel processing, are given in Table I. The model LM3 presents the best spatial efficiency with a scaling factor $k=3$, followed by $B M 1$, with the average scaling factor $k=4$. The model $B M 2$ presents the worse comparative efficiency, $k=8$, due to the fact that besides the ancillae qubits, every computational qubit is encoded in four physical qubits.

\begin{tabular}{|c|c|c|}
\hline architecture & $C_{\mathcal{H}_{\text {sing }}}^{*}(\mathcal{U})$ & time $(\mu \mathrm{s})$ \\
\hline$L M 3$ & {$[441.6(n+m)+92.0] J^{-1}$} & 10.7 \\
\hline$B M 2$ & $(403.2 n+134.4 m+1065.6) J^{-1}$ & 24.0 \\
\hline$B M 1$ & $(28.2 n+9.4 m+15.6) J^{-1}$ & 0.25 \\
\hline
\end{tabular}

TABLE II: Additional cost for the realization of one ( $n=0$, neglecting all constant terms) and two computational qubit gates for the three models as a function of the Ising coupling strength $J$. The last column reports the associated time for $J=50 \mathrm{MHz}$.

Table II shows the results of the calculation of the time performance of general two-qubit computational gates i.e., gates where both computational qubits may be affected, for each architecture implemented under the Ising $\mathcal{H}_{\text {Ising }}$ interaction Hamiltonian. In this table, $C_{\mathcal{H}_{\text {Ising }}}^{*}(\mathcal{U})$ represents the optimal additional time spent in the execution of the arbitrary twoqubit gate $\mathcal{U}$ under the coupling $\mathcal{H}_{\text {Ising }}$, and using GCQC protocols. When the asterisk is absent, this quantity has the same 
meaning, but for LCQC arrays. This initial choice of an Ising type of interaction is motivated by the following: i) given the heterostructural nature of the GCQC models, a non-resonant interaction is expected to be the most efficient interaction; ii) results for optimal simulation of three qubit quantum gates has only been developed for Ising type of interactions $[15,18]$.

There are two time scale factors in Table II: $g_{1}$ and $g_{2}$, which are associated to the two transportation processes represented by $n$ and $m$ respectively. The variable $m$ is associated with the localization of one of the qubits involved in the computational gate by the control unit, and $n$ to the transportation of the information between the two computational qubits involved in the gate execution. The model $B M 1$ exhibits the best time efficiency with factors $g_{1}=28.2$ and $g_{2}=9.4$. The fact that, for $B M 2$ and $B M 1$, general two-qubit computational gates have to be realized by the action of two controlled gates, triples the magnitude of the factor $g_{1}$.

The additional times $C_{\mathcal{H}_{\text {Ising }}}^{*}(\mathcal{U})$ are shown in Table II. For the purpose of illustration, specific times were calculated for the case $m=0$ and $n=1$ (control unit and computational qubits adjacent to each other) under coupling strength $J=$ $50 \mathrm{MHz} \approx 33 \mathrm{neV}$, which is a common order of magnitude for magnetic dipolar interactions in proposals such as fullerenebased electron spin quantum computers [20,21]. The results are shown in Table II. For the three models, the one with lesser additional time spent is $B M 1$, with a specific value of $0.25 \mu \mathrm{s}$. Just for the sake of comparison, this is equivalent to 5.3 times the optimal time required in the direct realization of the $S W A P$ gate, which performs the exchange of quantum states between qubits [22], and which, for $J=50 \mathrm{MHz}$, is $47.1 \mathrm{~ns}$, as reported in Table IV. In contrast, the greater additional time, due to $B M 2$, presents an extra cost equivalent to 509 times the direct application of the SWAP gate. In the case of $B M 2$ and $B M 1$ the same rates are found in comparison with the direct execution of the $C N O T$ gate, given that $C_{\mathcal{H}_{\text {Ising }}}^{*}(S W A P)=$ $3 C_{\mathcal{H}_{\text {Ising }}}^{*}(C N O T)$ and $C_{\mathcal{H}_{\text {Ising }}}(S W A P)=3 C_{\mathcal{H}_{\text {Ising }}}(C N O T)$. The first equation comes from the observation that for $B M 1$ and $B M 2$ the additional computational time of general two-qubit gates, where both qubits are affected, triple that of a two-qubit controlled gate. The latter equation is directly deduced from Table IV.

\begin{tabular}{|c|c|}
\hline model $B M 1$ & time $(\mathrm{ps})$ \\
\hline$(14.1 n+4.7 m+15.6) J_{F}^{-1}$ & 13.3 \\
\hline
\end{tabular}

TABLE III: Additional cost for the realization of one $(n=0, m=1)$ and two computational qubit gates in the model $B M 1$ for the Förster coupling strength $J_{F}$. The second column gives the required time for $J_{F}=1.5 \mathrm{THz}$.

Next, we compute the optimal additional time for general computational two-qubit gates for the case of a generic physical system where the qubits interact via the Förster coupling: $J_{X Y} \equiv J_{F}, J=0$ in Eq. (6) [23-26]. We do so for the model $B M 1$. The calculation for the models $L M 3$ and BM2 remains an open question due to the fact that optimal simulation for three qubit quantum gates under Förster interaction is still, hitherto, an unsolved problem. The $J_{F}$ coupling appears in many different physical systems, ranging from nanostructures such as quantum dots and wells [23-26] through to biomolecular systems [27-29]. The first column of Table III shows the general result associated to an arbitrary coupling intensity $J_{F}$. The last column shows the result for $J_{F}=1.5 \mathrm{THz} \approx 1 \mathrm{meV}$, which is a representative estimate for exchange interactions between quantum dots [23-26]. In this case, the parameters of "transport" $g_{1}$ and $g_{2}$ decrease by a factor of one half, expressing the fact that the Förster interaction is a more efficient interaction for energy transfer.

\begin{tabular}{|c|c|c|}
\hline $\mathcal{U}$ & $C_{\mathcal{H}_{\text {sing }}}(\mathcal{U})$ & time $(\mathrm{ns})$ \\
\hline SWAP & $(3 \pi / 4) J^{-1}$ & 47.1 \\
\hline$C N O T$ & $(\pi / 4) J^{-1}$ & 15.7 \\
\hline $\mathcal{U}$ & $C_{\mathcal{H}_{F}}(\mathcal{U})$ & time $(\mathrm{fs})$ \\
\hline SWAP & $(3 \pi / 8) J_{F}^{-1}$ & 775 \\
\hline$C N O T$ & $(\pi / 4) J_{F}^{-1}$ & 516 \\
\hline
\end{tabular}

TABLE IV: General and specific temporal costs for the direct execution of the SWAP and CNOT quantum gates under the i) Ising and ii) Förster interaction Hamiltonians. The last column is computed for couplings i) $J=50 \mathrm{MHz}(33 \mathrm{neV})$ and ii) $J_{F}=1.5 \mathrm{THz}(1 \mathrm{meV})$, respectively.

To illustrate this point, in Table IV we show the optimal time for the execution of the two-qubit logic gates SWAP and $C N O T$. The SWAP gate is involved in the transportation of the computational qubits along the quantum register in $B M 1$. The CNOT gate applies the quantum NOT gate (quantum version of the classical NOT gate) conditioned by the computational state of its neighbouring qubit. We see from Table IV that $C_{\mathcal{H}_{F}}(S W A P)$ is one half times $C_{\mathcal{H}_{\text {Ising }}}(S W A P)$ for $J=J_{F}$. The factor 15.6 of Table III , which is independent of the processes of transportation, remains the same as that of Table II because this only contains controlled gates which have the same generic value for both, Ising and Förster, couplings (see Table IV).

The additional time spent in the execution of a general twoqubit gate between neighbouring computational qubits under the Förster coupling $J_{F}=1.5 \mathrm{THz}$ is approximately 17.2 times the optimal time required for the execution of the SWAP gate and 8.6 times the one required in the direct execution of the CNOT gate, under the same coupling conditions (see Table IV). The difference of five orders of magnitude in the optimal time required for the direct two-qubit gate execution between systems interacting under Ising and Förster coupling relies on the difference in their corresponding coupling intensities as taken from some representative systems such as fullerene-based electron spin quantum computers $[20,21]$ and systems of quantum dots coupled via exchange interactions $[23,26]$.

Up to now we have compared the spatial scalability and temporal efficency of the three architectures. A fundamental requirement for any architecture, in order to support quantum computation is its ability to implement quantum error correction over its computational and ancillae qubits. The following 
section is dedicated to a discussion of the implementation of quantum error correction in the three GCQC models presented above.

\subsection{Quantum Error Correction}

The overall additional resources required for these architectures have important effects on their ability to implement quantum error correction codes (QECCs) [31]. In fact, faulttolerant thresholds impose minimal values for coupling intensities between adjacent qubits. Calculation of these thresholds depends on the specific architecture and error correction strategies to be used [32], and some key observations can be made in this respect. Quantum error correction in GCQC models can be divided into two subproblems: i) quantum error correction of computational qubits and ii) classical error correction of ancillae qubits. The problem of quantum correction of computational qubits may be solved by QECCs [22] but the implementation of QECCs in GCQC models is not a straightforward process. For example, contrary to what is suggested in Ref. [33], computational qubits in the model BM2 cannot be corrected by QECCs because their architectural encoding doesn't match with any QECC. To illustrate this, let us try to correct one computational qubit from a bit-flip error using the stabilizers formalism [22].

(a)

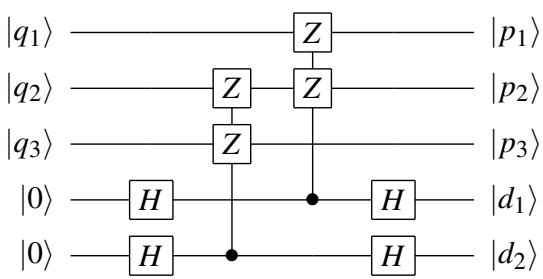

(b)

\begin{tabular}{|c|c|c|}
\hline input & diagnostic & output \\
\hline$\{|000\rangle,|111\rangle\}$ & $|00\rangle$ & $\{|000\rangle,|111\rangle\}$ \\
\hline$\{|00 \mathbf{1}\rangle,|11 \mathbf{0}\rangle\}$ & $|01\rangle$ & $\{|000\rangle,|111\rangle\}$ \\
\hline$\{|\mathbf{1 0 0}\rangle,|\mathbf{0 1 1}\rangle\}$ & $|10\rangle$ & $\{|000\rangle,|111\rangle\}$ \\
\hline$\{|0 \mathbf{1 0}\rangle,|1 \mathbf{0 1}\rangle\}$ & $|11\rangle$ & $\{|000\rangle,|111\rangle\}$ \\
\hline
\end{tabular}

FIG. 4: (a) Error diagnostic code for the bit-flip one qubit error. (b) Input, diagnostic, and output for the QECC.

Figure 4 shows the diagnostic circuit using the set $\left\{Z_{2} Z_{3}, Z_{1} Z_{2}\right\}$ as the stabilizer generators. One computational qubit is encoded into three physical qubits in the following form: $|0\rangle_{c} \equiv|000\rangle$ and $|1\rangle_{c} \equiv|111\rangle$. This circuit detects and therefore corrects all one bit-flip errors. Figure 4(b) shows the set (input) of correctable encoded qubits. However, in order to implement the bit-flip QECC in $B M 2$, one has to encode each qubit, $\left|q_{i}\right\rangle$, into four physical qubits following the allocation given by the architecture: $|0\rangle \equiv|\uparrow \uparrow \downarrow \downarrow\rangle$, and $|1\rangle \equiv|\downarrow \downarrow \uparrow \uparrow\rangle$. This leads to a total of twelve physical qubits per computational qubit encoded. It is easy to check that any one phys- ical qubit bit-flip error over an encoded computational qubit doesn't belong to the set of correctable encoded qubits depicted in Fig. 4(b). The same argument is valid to show the inability of $B M 2$ to correct computational qubits through any other QECC. In fact, the only cases where QECC is possible in QC models with architectural encoded qubits is when this encoding matches that of the QECC.

For the other models analyzed here, QECC is possible as long as a degree of parallelism of at least $O(\log (N))$ is feasible [34]. This fact makes the design of complementary strategies allowing parallel QECC processing in LM3 and BMI a must. A strategy has already been put forward for BM1 [32], using periodically distributed "switching stations" where control units are activated and deactivated, except one, which is always activated and is used for the computation of the actual algorithm. Parallel processing on LM3 hasn't yet been explored.

The second issue, that of ancillae qubit correction, is an involved problem. As the aim of error correction on ancillae is to keep the ancillae qubits in their ground state, $|0\rangle$, correction is in this case to be carried out by dissipative operations over the register, also known as "resetting". To achieve this, it has recently been proposed to consider a third level for each qubit, such that it could be populated from either of the states $|0\rangle$ and $|1\rangle$, and would decay in a dissipative way into one particular state, say $|0\rangle[12,32]$. Aside from this, one more question remains: how to distinguish ancillae from computational qubits? In the case of $L M 3$, computational qubits are naturally distinguished by the species they are, in a given time step. In $B M 1$, this is not the case, because there are always ancillae qubits in the same species where the computational qubits are. A second alternative is to use the control unit to localize ancillae corrections, an effective strategy but less efficient compared to the one for LM3, where parallelism is maximal. Thus, parallel processing is necessary for QECC implementation in $L M 3$ and $B M 1$, and the physical requirement of a third energy level for each qubit will certainly be a common requirement in $B M 1$ and $L M 3$ in order to allow parallel QECCs, if strategies such as those proposed in Refs. [12, 32] are to be implemented.

\section{CONCLUSIONS}

From the analysis given above, we conclude that $B M 1$ presents the best overall efficiency in spatial and temporal resources, without considering error correction of computational and ancillae qubits. The hierarchy of time efficiency of two-qubit gate execution was determined assuming an Ising type of interaction. In the case of $B M 1$, it was also calculated for systems coupled via the Förster interaction. We also conclude that only $B M 1$ and $L M 3$ remain as feasible candidates for QECC implementation, discarding BM2 since their computational qubits have an architectural encoding which doesn't match with any QECC. 


\section{ACKNOWLEDGEMENTS}

We are grateful to COLCIENCIAS for financial support under research contracts 1106-14-17903 and 1106-45-221296, and the scientific exchange program PROCOL (DAADColciencias).
[1] R. P. Feynman, "There is plenty of room at the bottom", talk given by Richard Feynman on December 29, 1959, at the meeting of the American Physical Society at Caltech.

[2] R. P. Feynman, Int. J. Theor. Phys. 21, 467 (1982)

[3] D. Deutsch, Proc. R. Soc. Lond. A 400, 97 (1985); ibid. 425 , 73 (1989).

[4] P. W. Shor, Proceedings of the 35th symposium on Foundations of Computer Science, IEEE Computer Society Press, pp. 124 (1994).

[5] C. Zalka, Proc. R. Soc. Lond. A 454, 313 (1998).

[6] R. Raussendorf and H. J. Briegel, Phys. Rev. Lett. 86, 5188 (2000).

[7] E. Farhi, J. Goldstone, S. Gutmann, J. Lapan, and A. Lundgren, Science 292, 472 (2001)

[8] C. A. Perez-Delgado and D. Chueng, e-print arxiv: quant$\mathrm{ph} / 0508164$.

[9] A. M. Steane, Nature 399, 124 (1999).

[10] D. P. DiVincenzo. Phys. Rev. A 51, 1015 (1995).

[11] S. C. Benjamin, Phys. Rev. Lett. 88, 017904 (2002).

[12] S. C. Benjamin, Phys. Rev. A 61, 020301 (2000).

[13] S. Lloyd, Science 261, 1569 (1993).

[14] G. Vidal, K. Hammerer, and J. I. Cirac, Phys. Rev. Lett. 88 237902 (2002).

[15] N. Khaneja, R. Brockett, and S. J. Glaser, Phys. Rev. A 65, 032301 (2002).

[16] N. Khaneja, R. Brockett, and S. J. Glaser, Phys. Rev. A 63, 032308 (2001).

[17] K. Hammerer, G. Vidal, and J. I. Cirac, Rev. A 66, 062321 (2002).

[18] N. Khaneja, B. Heitmann, A. Spoerl, H. Yuan, T. SchulteHerbrueggen, and S. J. Glaser, e-print arXiv: quant$\mathrm{ph} / 0605071$.

[19] S. C. Benjamin, B. Lovett, and J. H. Reina, Phys. Rev. A 70,
060305(R) (2004).

[20] W. Harneit, Phys. Rev. A 65, 032322 (2002).

[21] S. C. Benjamin, A. Ardavan, A. Briggs, D. A. Britz, D. Gunlycke, J. Jefferson, M. Jones, D. F. Leigh, B. W. Lovett, A. N. Khlobystov, S. A. Lyon, J. Morton, K. Porfyrakis, M. R. Sambrook and A. M. Tyryshkin, J. Phys.: Condensed Matter 18, 867 (2006)

[22] M. A. Nielsen and I. L. Chuang, Quantum Information and Computation, Cambridge University Press (2000).

[23] J. H. Reina, L. Quiroga, and N. F. Johnson, Phys. Rev. A 62, 012305 (2000).

[24] B. W. Lovett, J. H. Reina, A. Nazir, B. Kothari, and A. Briggs, Phys. Lett. A 315, 136 (2003).

[25] B. W. Lovett, J. H. Reina, A. Nazir, and A. Briggs, Phys. Rev. B 68, 205319 (2003).

[26] A. Nazir, B. Lovett, S. Barrett, J. H. Reina and A. Briggs, Phys. Rev. B 71, 045334 (2005).

[27] T. Renger, V. May, and O. Kühn, Phys. Rep. 343, 137 (2001).

[28] G. S. Engel, T. R. Calhoun, E. L. Read, T.-K. Ahn, T. Mancal, Y.-C. Cheng, R. E. Blankenship, and G. R. Fleming, Nature 446, 782 (2007).

[29] H. Lee, Y.-C. Cheng, and G. R. Fleming, Science 316, 1462 (2007).

[30] J. H. Reina, L. Quiroga and N. F. Johnson, Phys. Rev. A 65, 032326 (2002).

[31] A. M. Steane, Phys. Rev. Lett. 77, 793 (1996).

[32] A. Kay, e-print Arxiv: quant-ph/0702239.

[33] A. Bririd, S. C. Benjamin, and A. Kay, e-print Arxiv: quant$\mathrm{ph} / 0308113$.

[34] D. Aharonov and M. Ben-Or, Proc. of the 29th Annual ACM Symposium on Theory of Computing (STOC) (1997). 